Review

\title{
Research progress on the impact of radiation on TKI resistance mechanisms in NSCLC
}

\author{
Hongqing Zhuang ${ }^{\bowtie}$ \\ Department of Radiation Oncology, Peking University Third Hospital, Beijing, China \\ $\triangle$ Corresponding author: Peking University Third Hospital, 49 North Garden Road, Haidian District, Beijing 100191, P R China. Tel.: +86-010-82266699. E-mail \\ address: hongqingzhuang@163.com \\ (c) Ivyspring International Publisher. This is an open access article distributed under the terms of the Creative Commons Attribution (CC BY-NC) license \\ (https://creativecommons.org/licenses/by-nc/4.0/). See http://ivyspring.com/terms for full terms and conditions.
}

Received: 2018.03.29; Accepted: 2018.07.23; Published: 2018.10.01

\begin{abstract}
Resistance to tyrosine kinase inhibitor (TKI) therapy is often accompanied by various genetic alterations, and radiation is an important weapon for changing the DNA of tumor cells. In radiotherapy combined with TKI therapy for non-small cell lung cancer (NSCLC), the two treatment strategies affect and interact with each other, resulting in complex tumor resistance mechanisms. Accordingly, tumor progression management after radiotherapy combined with TKI therapy should be different from that after TKI therapy alone. However, current clinical practice is entirely based on the resistance mechanisms of simple TKI therapy. Therefore, it is imperative to investigate the impact of radiation on the mechanism of TKI resistance. However, due to the complexity of the resistance mechanisms under the combined effect of both therapies, such studies remain extremely challenging and time-consuming.
\end{abstract}

Key words: radiation; radiotherapy; TKI; secondary resistance

\section{Introduction}

Radiotherapy combined with TKI therapy has been increasingly applied for the treatment of lung cancer [1-5]. However, treatment for patients with disease progression was mostly based on resistance after TKI therapy alone [6]. As a powerful factor of DNA damage, radiation has the ability to alter Deoxyribonucleic acid (DNA) in tumor cells, leading to the development of radiation resistance. Radiation resistance may hybridize with TKI secondary resistance mechanisms, thereby affecting the TKI resistance mechanism. At the same time, radiation itself may also alter TKI resistance genes and further affect the TKI resistance mechanism. Thus, radiation may have a great impact on TKI resistance, and the investigation of this effect is important for patients undergoing clinical treatment after radiotherapy combined with TKI therapy. This paper reviews and summarizes the relevant literature on the study objective.

\section{TKI resistance mechanisms}

\subsection{Alteration of the gene on the target site}

The long-term effects of TKI on epidermal growth factor receptor (EGFR) target can lead to a loss or reduction in the binding force between TKI and the binding site of the target molecule. This in turn reduces the efficacy of TKI therapy and causes drug resistance. For example, T790M is a site in the EGFR exon 20, and long-term TKI treatment may cause point mutation at this site, thereby changing the crystal structure of the binding pocket for adenosine triphosphate (ATP) in the kinase domain and so that the binding of TKI with the kinase domain is blocked. In addition, mutation L858R together with T790M may enhance the affinity for ATP compared to that of L858R alone. Additionally, TKI is an inhibitor of ATP competitive kinase, which leads to a lower binding rate of TKI and the kinase domain, and result in the secondary resistance [7-10]. 


\subsection{Alteration of the downstream gene of the target site}

The efficacy of TKI requires signal transduction to the nucleus via downstream pathway of the target site. An abnormality of any site in the downstream pathway could have impacted the efficacy of TKI. For example, K-ras is a key gene in the downstream portion of the EGFR signaling pathway, and the mutated K-ras gene can directly activate the (mitogen-activated protein kinase, MAPK) signaling pathway without activating the upstream EGFR pathway, leading to the proliferation and metastasis of tumor cells. K-ras gene mutation is another important molecular mechanism of primary EGFR-TKI resistance [11, 12]. Additionally, a change in any site in the downstream EGFR pathways, similar to K-ras, affects the TKI efficacy and causes resistance.

\subsection{Activation or amplification of alternative pathways}

Pathways are complex networks. If one pathway is blocked, the remaining associated alternative pathways may be activated to maintain the growth and proliferation of tumor cells, i.e., when the EGFR-RAS-MAPK pathway has been blocked by TKI for an extended period of time, alternative pathways maybe activated. The most typical alternative is the PI3K-AKT-mTOR pathway. When EGFR is blocked, the phosphorylation levels of AKT and mTOR are enhanced, and the activity of the PI3K-AKT-mTOR pathway is significantly increased, there by establishing TKI resistance [13-15]. Another example is C-MET, when C-MET is amplified, the downstream PI3K-AKT-mTOR pathway may also be activated, thereby triggering TKI resistance $[16,17]$. Thus, this is similar to a river, wherein if the main river is blocked, the formation of tributaries is inevitably given sufficient time. Alternative pathways are also important mechanisms of TKI resistance.

The mechanisms of resistance in tumor cells are extremely complex, and many mechanisms still remain unclear. In addition to the above three mechanisms, there are other mechanisms influencing the formation of TKI resistance, such as the conversion between different pathological types as well as other drug-related pathways. These mechanisms remain poorly understood.

\section{Effects of radiation on genetic alterations}

\subsection{Radiation changes tumor-associated genes}

Radiation mainly targets DNA, and therefore, radiation is an important weapon for altering genes.
Previous studies have demonstrated the effects of radiation on DNA damage repair, the expression of cell cycle proteins and apoptosis-related proteins, and the growth and proliferation of tumor cells [18-20]. These genes may be cross-linked with EGFR pathway, thereby affecting TKI resistance and the efficacy of treatment after the development of resistance. However, due to the complexity of the tumor pathways, further research in this area is expected.

\subsection{Changes in radiation sensitivity-related genes during irradiation cause radiation resistance}

The radiation sensitivity of tumor cells is different from that of normal cells, and several genes are involved in this sensitivity. For example, when EGFR is highly expressed in tumor cells, the tumor tends to have a high level of resistance to radiation therapy. When radiation acts on the tumor cells, EGFR can enter the nucleus by a coupling process. In this process, $\mathrm{Ku} 70 / 80$ and phosphatase- 21 can enter the nucleus together with EGFR, resulting in an increase in DNA-PK activity. This subsequently increases the radiation resistance of the tumor cells[21,22]. In addition, cell lines with KRAS mutations may also regulate the expression of EGFR ligands, thus affecting the PI3K-AKT pathway and causing radiation resistance[23]. In fact, the survival of cells with different expression levels of sensitive genes following irradiation involves the process of killing tumor cells by radiation and the process of self-selection in tumor tissues under external conditions, thus establishing radiation-resistant cells. The genetic characteristics of radiation-resistant cells may also have an impact on the resistance and efficacy of TKI.

\section{The interaction of radiation-related genetic alterations and TKI resistance mechanisms}

\subsection{Effect of EGFR mutation on radiation sensitivity}

Previous studies have shown that the radiation sensitivity of various lung cancer cell lines with EGFR mutations was higher than that of the wild-type [24]. In addition, the results of a clinical study also suggested that when the lung cancer patients with brain metastasis underwent whole-brain radiotherapy, EGFR mutation was an independent factor that influenced the clinical response[25]. Further research demonstrated that the radiation sensitivity of NSCLC with EGFR mutation is closely related to its non-homologous end joining (NHEJ) dysfunction. After ionizing radiation, EGFR mutant 
cell lines and human bronchial epithelial cells (HBECs) with ectopic mutation in the EGFR gene could not effectively conduct EGFR nuclear translocation. Then the EGFR was unable to bind to the DNA-PK repair protein, thus greatly diminishing the DNA damage repair ability of the NHEJ pathway and enhancing radiation sensitivity [26]. The Ras/Raf/MEK/ERK and PI3K/AKT pathways on which the mutant EGFR is dependent are important means of repair via homologous recombination (HR); however, no relevant research on the relationship between EGFR mutations and HR has been reported yet. In addition, under radiation, the proteins for DNA damage repair (e.g., DNA-PKcs and Rad51) may also differ in their expression level or function [27]. All these potential mechanisms require further study.

\subsection{Impact of radiation on TKI resistance-related genes}

The altered expression and mutation of TKI resistance-related genes by radiation is an important aspect of the effect of radiation on TKI resistance. Studies have shown that for the primary mechanism of TKI resistance, radiation can decrease the rate of T790M mutation, thereby reducing the formation of TKI resistance [28]. At the same time, radiation also influences on the amplification of c-MET, which is another important mechanism of TKI resistance. Studies have shown that radiation can increase the expression of c-MET and activate the downstream pathways of the c-MET protein, there by maintaining the growth and proliferation of tumor cells under the effect of TKI [29]. However, to date, studies on the impact of radiation on TKI resistance-related genes are lacking. Most studies of resistance mechanisms have been confined to the field of medical oncology. The alteration of resistant genes by radiation requires further exploration.

\subsection{The presence of cross-signaling pathways for radiation and $\mathrm{TKI}$ resistance}

Alterations in signaling pathways are a common means by which radiation and TKI can affect the resistance and survival of tumor cells. Additionally, in a complex network of signaling pathways, multiple cross-signaling pathways exist between radiation and TKI resistance. Many studies have shown that the long-term application of TKI could activate the PI3K-AKT-MTOR pathway, resulting in secondary TKI resistance. At the same time, under radiation, the phosphorylation level of the PI3K-AKT-MTOR pathway was significantly increased, thereby enhancing the activity of the pathway [30-32]. Thus, under the effect of both treatments, this pathway is more easily activated, there by producing TKI resistance. Therefore, the PI3K-AKT-MTOR pathway is an important alternative means for cell signaling if the MAPK pathway is blocked by TKI, and this mechanism represents to be one of the principal contradictions in the resistance of tumor cells under radiotherapy combined with TKI therapy (Figure 1). The complex networks of signaling pathways provide natural conditions for the activation of resistance pathways in tumor cells under the combined effect of radiation and TKI. This acts as an important mechanism of resistance in cells under the combined effect of radiation and TKI.

\subsection{Influence of the schedule of radiotherapy and TKI application on the mechanisms of drug resistance}

In clinic practice, the key problem about the combination of TKI and radiotherapy was the combination mode especially for optimal timing of these therapeutic regimens like in treatment of thoracic radiation and brain metastases. Magnuson WJ et al. demonstrated that the use of upfront RT or upfront EGFR-TKI is associated with different OS in patients with EGFR-mutant NSCLC who develop brain metastases [33,34]. It may be that these mechanisms affect the combination schedule and effect. Similarly, if radiotherapy was used first, the tumor gene may have changed, and then TKI was applied. The mechanism of drug resistance was also based on the gene after radiotherapy. By the same token, if patients first used TKI and then radiotherapy, or used together, the final resistance mechanism may also be different. In addition, the mechanism of drug resistance was a dynamic process. The schedule of participation should have an impact on the drug resistance mechanism after radiotherapy combined with TKI treatment.

\section{Problems}

\subsection{Identifying the breakthrough point in the study of the impact of radiation on TKI resistance mechanisms}

The EGFR pathway network extends in all directions, and changes in the resistance mechanisms of the tumors under the combined effects of radiation and TKI are cross-linked and complex such that a tumor cell may harbor various mechanisms of resistance [33]. Therefore, it is impossible to study all mechanisms. The breakthrough point in the study of the impact of radiation on TKI resistance mechanisms can be identified only by identifying the principal contradiction of the tumor resistance mechanisms under the combined effect so that the targeted studies 


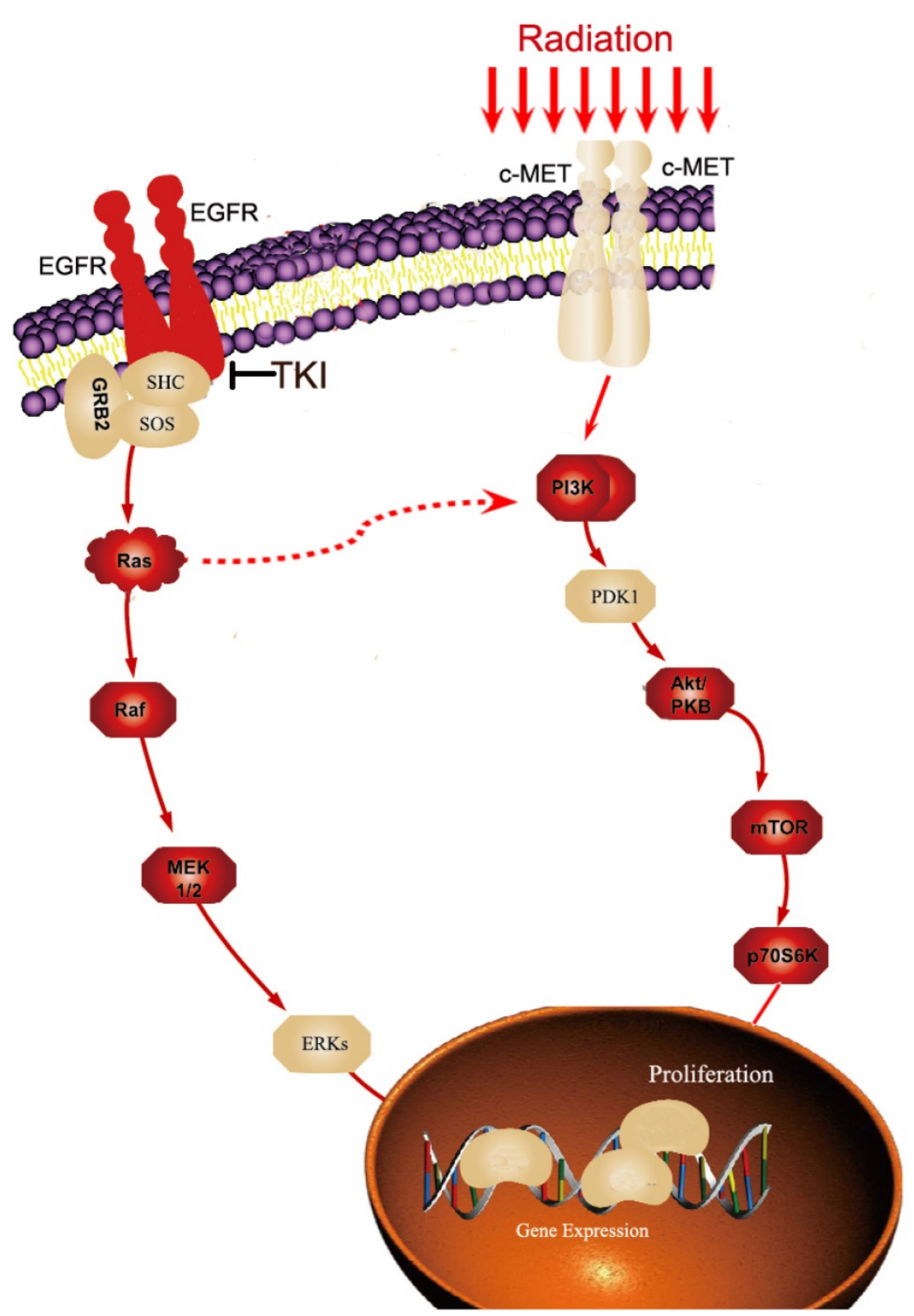

Figure 1: The example of cross-talk pathways for radiation and TKI resistance. Long-term application of TKI could activate PI3K-AKT-MTORpathway. At the same time, under radiation, the c-MET expression and the phosphorylation level of the PI3K-AKT-MTOR pathway were significantly increased, thereby enhancing the activity of the pathway. Therefore, the PI3K-AKT-MTOR pathway is an important alternative means for cell signaling if MAPK pathway is blocked by TKI.

can be more efficient. This requires extensive preliminary research, and pre-screening in these preliminary studies will contribute to reasonable judgments.

\subsection{Detection of the resistance mechanisms under the combined effect}

First, for either TKI or radiation, the resulting genetic alteration of the tumor cells has its own independent process. The changes in its mechanism under the combined effect may be different from those under the single treatment. Therefore, accurate identification of the temporal effects of gene alterations under the combined effects of the two treatments is one of the difficulties in determining the resistance mechanisms. Second, the current detection of resistance mechanisms requires the accumulation of a large number of specimens to obtain a relatively precise conclusion. However, in clinical practice, patients receiving combined therapy often require long-term treatment, and thus, re-sampling for pathological detection at the disease progression site is often difficult, leading to a greater challenge in the detection of resistance mechanisms. Third, the selection of the detection methods is also a matter of concern. If the specimens are not easily available, blood tests appear to be the ideal alternative. However, the sensitivity of blood tests may introduce a new issue to the study of resistance mechanisms [34-36].

\subsection{Intervention for preventing resistance}

The purpose of investigating resistance mechanisms is to provide intervention. The resistance mechanism of radiation combined with TKI is multifaceted, and thus, the efficacy of a single treatment maybe poor. However, the combination of more than one treatment could lead to additional genetic alterations and further drug resistance [37]. Therefore, identifying the main mechanism of resistance is the key to determine the breakthrough point for intervention. In addition, the introduction of novel ideas, critical evaluation of the resistance -intervention- more resistance concepts and the search of additional treatments unrelated to TKI resistance mechanisms might be a more reasonable intervention options. For example, after resistance occurs following the combination of radiation and TKI, a valid option may be considered as immune therapy, so that the tumor cells should never undergo to other treatments.

In summary, radiation is an important means of genetic alteration, and resistance is the only final outcome of the application of TKI. The increasing use of radiotherapy combined with TKI in NSCLC has led 
to an urgent need to study the impact of radiation on TKI resistance mechanisms. However, the effects of radiation and TKI on tumor cells can be interactive and are highly complex. Thus, extensive exploration is needed on the impact of radiation on TKI resistance mechanisms, which lead to a greater requirement for basic and clinical research in this area. We believe that with the gradual expansion of basic research and the accumulation of the clinical data, the impact of radiation on TKI resistance mechanisms becomes increasingly clear. Interventions for resistance after radiotherapy combined with TKI becomes more accurate, and radiotherapy combined with TKI will have a broader application potential.

\section{Acknowledgement}

This Paper was supported by National Natural Science Foundation of China (81301925) and Clinical key project of Peking University Third Hospital (BYSY2017030).

\section{Competing Interests}

The authors have declared that no competing interest exists.

\section{References}

1. Zhuang H, Zhao X, Zhao L, Chang JY, Wang P. Progress of clinical research on targeted therapy combined with thoracic radiotherapy for non-small-cell lung cancer. Drug Des Devel Ther. 2014:8:667-75.

2. Zhuang H, Yuan Z, Wang J, Zhao L, Pang Q, Wang P. Phase II study of whole brain radiotherapy with or without erlotinib in patients with multiple brain metastases from lung adenocarcinoma. Drug Des Devel Ther. 2013;7:1179-86.

3. Zhuang $\mathrm{H}$, Wang J, Zhao L, et al. The theoretical foundation and research progress for WBRT combined with erlotinib for the treatment of multiple brain metastases in patients with lung adenocarcinoma. Int J Cancer. 2013; 133(10):2277-2283.

4. Wang J, Xia TY, Wang YJ et al. Prospective study of epidermal growth factor receptor tyrosine kinase inhibitors concurrent with individualized radiotherapy for patients with locally advanced or metastatic non-small-cell lung cancer. Int J Radiat Oncol Biol Phys. 2011;81(3):e59-65.

5. Chang $\mathrm{CC}, \mathrm{Chi} \mathrm{KH}, \mathrm{Kao} \mathrm{SJ}$, et al. Upfront gefitinib/erlotinib treatment followed by concomitant radiotherapy for advanced lung cancer: a mono-institutional experience. Lung Cancer. 2011;73(2):189-94.

6. Yang JJ, Chen HJ, Yan HH, et al. Clinical modes of EGFR tyrosine kinase inhibitor failure and subsequent management in advanced non-small cell lung cancer. Lung Cancer. 2013;79(1):33-9.

7. Kobayashi S, Boggon TJ, Dayaram T, et al. EGFR mutation and resistance of non-small-cell lung cancer to gefitinib. N Engl J Med, 2005, 352(8): 786-792.

8. Yun $\mathrm{CH}$, Mengwasser KE, Toms AV et al. The T790M mutation in EGFR kinase causes drug resistance by increasing the affinity for ATP. Proc Natl Acad Sci USA, 2008, 105(6): 2070-2075

9. Forde PM, Ettinger DS. Managing acquired resistance in EGFR-mutated non-small cell lung cancer. Clin Adv Hematol Oncol. 2015·13(8):528-32.

10. Villadolid J, Ersek JL, Fong MK, Sirianno L, Story ES. Management of hyperglycemia from epidermal growth factor receptor (EGFR) tyrosine kinase inhibitors (TKIs) targeting T790M-mediated resistance. Transl Lung Cancer Res. 2015;4(5):576-83.

11. Uramoto H, Shimokawa H, Hanagiri T, Kuwano M, Ono M. Expression of selected gene for acquired drug resistance to EGFR-TKI in lung adenocarcinoma. Lung Cancer. 2011·73(3):361-5.

12. Califano R, Landi L, Cappuzzo F. Prognostic and predictive value of K-RAS mutations in non-small cell lung cancer. Drugs. 2012;72 Suppl 1:28-36.

13. Gadgeel SM, Wozniak A. Preclinical rationale for PI3K/Akt/mTOR pathway inhibitors as therapy for epidermal growth factor receptor inhibitor-resistant non-small-cell lung cancer. Clin Lung Cancer. 2013;14(4):322-32.

14. Fumarola C, Bonelli MA, Petronini PG, Alfieri RR. Targeting $\mathrm{PI} 3 \mathrm{~K} / \mathrm{AKT} / \mathrm{mTOR}$ pathway in non small cell lung cancer. Biochem Pharmacol. 2014;90(3):197-207.
15. Sun Z, Wang Z, Liu X, Wang D. New development of inhibitors targeting the $\mathrm{PI} 3 \mathrm{~K} / \mathrm{AKT} / \mathrm{mTOR}$ pathway in personalized treatment of non-small-cell lung cancer. Anticancer Drugs. 2015;26(1):1-14

16. Passiglia F, Van Der Steen N, Raez L, et al. The role of cMet in non-small cell lung cancer resistant to EGFR-inhibitors: did we really find the target? Curr Drug Targets. 2014;15(14):1284-92.

17. Botting GM, Rastogi I, Chhabra G, Nlend M, Puri N. Mechanism of Resistance and Novel Targets Mediating Resistance to EGFR and c-Met Tyrosine Kinase Inhibitors in Non-Small Cell Lung Cancer. PLoS One. 2015;10(8):e0136155.

18. Zhuang HQ Sun J, Yuan ZY, et al. Radiosensitizing effects of gefitinib at different administration times in vitro. Cancer Sci. 2009;100(8):1520-5.

19. Peters LJ. Through a glass darkly: predicting the future of radiation oncology. Int J Radiat Oncol Biol Phys. 1995;31(2):219-25.

20. Kinsella TJ. Radiation oncology: today and tomorrow. Front Oncol. 2011;1:7.

21. Dittmann K, Mayer C, Fehrenbacher B, et al. Radiation2induced epidermal growth factor receptor nuclear import is linked to activation of DNA2 dependent protein kinase. J Biol Chem. 2005, 280: 31189-22.

22. Dittmann K, Mayer C, Rodemann HP, et al . Inhibition of radiation induced EGFR nuclear import by C225 (Cetuximab) suppresses DNA-PK activity. Radiother Oncol. 2005, 76:1572161.

23. Minjgee M, Toulany M, Kehlbach R, Giehl K, Rodemann HP. K-RAS(V12) induces autocrine production of EGFR ligands and mediates radioresistance through EGFR-dependent Akt signaling and activation of DNA-PKcs. Int J Radiat Oncol Biol Phys. 2011:81(5):1506-14.

24. Das AK, Sato M, Story MD, et al. Non-small-cell lung cancers with kinase domain mutations in the epidermal growth factor receptor are sensitive to ionizing radiation. Cancer Res. 2006:66(19):9601-8.

25. Gow $\mathrm{CH}$, Chien $\mathrm{CR}$, Chang $\mathrm{YL}$, et al. Radiotherapy in lung adenocarcinoma with brain metastases: effects of activating epidermal growth factor receptor mutations on clinical response. Clin Cancer Res. 2008;14(1):162-8.

26. Das AK, Chen BP, Story MD, et al. Somatic mutations in the tyrosine kinase domain of epidermal growth factor receptor (EGFR) abrogate EGFR-mediated radioprotection in non-small cell lung carcinoma. Cancer Res. 2007;67(11):5267-74.

27. Zhong $X$, Luo G, Zhou X, et al. Rad51 in regulating the radiosensitivity of non-small cell lung cancer with different epidermal growth factor receptor mutation status. Thorac Cancer. 2016;7(1):50-60.

28. Li J, Wu X, Wang Z, Shen Z, Sun N, Zhu X. Ionizing Radiation Reduces TKI Resistance Caused by T790M Mutation in NSCLC Cell Lines. Zhongguo Fei Ai Za Zhi. 2015;18(8):475-80.

29. Zhuang $\mathrm{HQ}$, Zhuang $\mathrm{H}, \mathrm{Bo} \mathrm{Q}$, et al. Experimental study on the regulation of erlotinib-induced radiosensitization with an anti-c-MET monoclonal antibody. Cancer Cell Int. 2014;14(1):109.

30. Zhuang HQ, Bo QF, Yuan ZY, Wang J, Zhao LJ, Wang P. The different radiosensitivity when combining erlotinib with radiation at different administration schedules might be related to activity variations in c-MET-PI3K-AKT signal transduction. Onco Targets Ther. 2013;6:603-8.

31. Sato $Y$, Ebara $T$, Sunaga N, Takahashi $T$, Nakano $T$. Interaction of radiation and gefitinib on a human lung cancer cell line with mutant EGFR gene in vitro. Anticancer Res. 2012;32(11):4877-81.

32. Contessa JN, Abell A, Valerie K, Lin PS, Schmidt-Ullrich RK. ErbB receptor tyrosine kinase network inhibition radiosensitizes carcinoma cells. Int J Radiat Oncol Biol Phys. 2006;65(3):851-8.

33. Magnuson $\mathrm{WJ}$, Lester-Coll $\mathrm{NH}_{\mathrm{H}} \mathrm{Wu}_{\mathrm{AJ}}$ et al Management of Brain Metastases in Tyrosine Kinase Inhibitor-Naïve Epidermal Growth Factor Receptor-Mutant Non-Small-Cell Lung Cancer: A Retrospective Multi-Institutional Analysis. J Clin Oncol. 2017;35(10):1070-1077.

34. Magnuson WJ, Yeung JT, Guillod PD, et al. Impact of Deferring Radiation Therapy in Patients With Epidermal Growth Factor Receptor-Mutant Non-Small Cell Lung Cancer Who Develop Brain Metastases. Int J Radiat Oncol Biol Phys. 2016 Jun 1;95(2):673-9.35.

35. Seshacharyulu P, Ponnusamy MP, Haridas D, Jain M, Ganti AK, Batra SK. Targeting the EGFR signaling pathway in cancer therapy. Expert Opin Ther Targets. 2012;16(1):15-31.

36. Sundaresan TK, Sequist LV, Heymach JV, et al. Detection of T790M, the Acquired Resistance EGFR Mutation, by Tumor Biopsy versus Noninvasive Blood-Based Analyses. Clin Cancer Res. 2016;22(5):1103-10.

37. Bordi P, Del Re M, Danesi R, Tiseo M. Circulating DNA in diagnosis and monitoring EGFR gene mutations in advanced non-small cell lung cancer. Transl Lung Cancer Res. 2015;4(5):584-97.

38. Zhuang HQ, Yuan ZY, Wang J, Wang P, Zhao LJ, Zhang BL. Research progress on criteria for discontinuation of EGFR inhibitor therapy. Onco Targets Ther. 2012; 5:263-70 